\title{
Heavily Si-doped InAs photoluminescence measurements
}

\author{
KaCPer Grodecki $^{1, *}$, Krzysztof Murawski ${ }^{1}$, Aleksandra Henig $^{1}$,

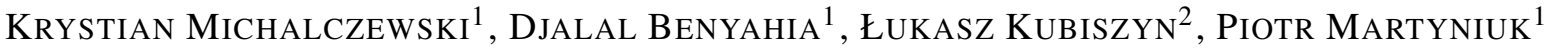 \\ ${ }^{1}$ Institute of Applied Physics, Military University of Technology, 2 Kaliskiego St., 00-908 Warsaw, Poland \\ ${ }^{2}$ Vigo System S.A., 129/133 Poznańska St., 05-850 Ożarów Mazowiecki, Poland
}

\begin{abstract}
In this paper, we present experimental results of photoluminescence for series of InAs:Si heavily doped samples, with doping level varying from $1.6 \times 10^{16} \mathrm{~cm}^{-3}$ to $2.93 \times 10^{18} \mathrm{~cm}^{-3}$. All samples were grown using MBE system equipped with a valved arsenic cracker. The measurements were performed in the temperature range of $20 \mathrm{~K}$ to $100 \mathrm{~K}$. Although the Mott transition in InAs appears for electron concentrations above $10^{14} \mathrm{~cm}^{-3}$, Burstein-Moss broadening of photoluminescence spectra presented in this article was observed only for samples with concentration higher than $2 \times 10^{17} \mathrm{~cm}^{-3}$. For the samples with lower concentrations two peaks were observed, arising from the band gap and defect states. The intensity of the defect peak was found to be decreasing with increasing temperature as well as increasing concentration, up to the point of disappearance when the Burstein-Moss broadening was visible.
\end{abstract}

Keywords: InAs; photoluminescence; Burstein-Moss; heavy doping

\section{Introduction}

InAs is a proper material for production of infrared photodetectors and infrared light emitting diodes working in $3 \mu \mathrm{m}$ to $5 \mu \mathrm{m}$ wavelength range [1-3]. Contrary to the other III-V materials, such as antimony compounds, InAs devices may operate at room temperature [4], which in turn, leads to the reduction of photodetector cost. Most of InAs photodiode heterostructures consist of undoped absorber and contacts made of p-type and strong n-type $\left(1 \times 10^{18} \mathrm{~cm}^{-3}\right)$ materials [58]. InAs of both p-type and n-type have been already studied in many works [9-11]. Therefore, the great number of InAs parameters, such as band gap energy as well as defect, donor, acceptor energies, have been determined [11, 12]. However, the characterization of heavily n-doped InAs is still not sufficient. Theoretical calculations showed that for doping higher than $10^{17} \mathrm{~cm}^{-3}$, Burstein-Moss shift and band gap peak broadening appear [13]. These phenomena may be described as an increased probability of indirect transitions, which in turn, leads to their appearance, contributing

*E-mail: kacper.grodecki@wat.edu.pl to the measured spectra [13]. This effect was also visible in experimental results presented by Gladkov et al. [14].

In this work, we present experimental results that confirm broadening of the band gap peak only for highly doped samples. Additionally observed effects are: the decrease of defect-induced peak at $35 \mathrm{meV}[11,15]$ and the decrease of defect peak intensity with increasing temperature. They may all be explained by the analysis of electron energy and momentum in highly doped InAs.

\section{Experimental}

The samples studied in this paper were grown on GaAs $\left(\begin{array}{lll}0 & 0 & 1\end{array}\right)$ substrate with $2^{\circ}$ offcut towards $\left\langle\begin{array}{lll}1 & 1 & 0\end{array}\right.$ by a solid source RIBER COMPACT $21 \mathrm{DZ}$ molecular beam epitaxy (MBE) system equipped with a valved arsenic cracker. The growth details have been reported in the literature [16]. The InAs layers were grown at $400{ }^{\circ} \mathrm{C}$, with an As/In flux ratio of 8.5. The growth rate was $0.26 \mu \mathrm{m} / \mathrm{h}$. The n-type doping was introduced by opening the shutter of $\mathrm{Si}$ effusion cell while growing InAs epilayers. The level of doping was calibrated by the temperature of $\mathrm{Si}$ effusion cell. Hall effect 
measurements using van der Pauw method were performed by ECOPIA Hall Measurements System, in order to assess the electrical parameters of the samples between $80 \mathrm{~K}$ and room temperature.

A high-resolution X-ray diffractometer of PANalytical X'Pert was utilized to evaluate the crystallographic properties of the samples. The $\mathrm{CuK} \alpha_{1}$ radiation $(\lambda \sim 1.5406 \AA)$ originating from a line focus was used. The X-ray beam was monochromatized by four bounce, Ge (l0 04 ) hybrid monochromator. The measurements were made in both $\omega$ and $2 \theta-\omega$ directions.

Basic parameters of the samples are presented in Table 1.

Table 1. Concentration and XRD Full Width at Half Maximum (FWHMs) for measured samples.

\begin{tabular}{lcc}
\hline $\begin{array}{c}\text { Sample } \\
{\left[\mathrm{cm}^{-3}\right]}\end{array}$ & $\begin{array}{c}\text { Concentration } \\
\text { FWHM of XRD } \\
\text { peak [arc sec] }\end{array}$ \\
\hline \hline A & $1.60 \times 10^{16}$ & 37 \\
B & $1.80 \times 10^{16}$ & 27 \\
C & $2.00 \times 10^{16}$ & 32 \\
D & $2.13 \times 10^{17}$ & 39 \\
E & $2.93 \times 10^{18}$ & 22 \\
\hline
\end{tabular}

Photoluminescence was measured using Vertex v70 FT-IR spectrometer in a step-scan mode, equipped with lock-in [17]. As an excitation source a $630 \mathrm{~nm}$ diode laser with mechanical chopper was used. During measurements, the samples were placed in a cryostat equipped with piezo table.

\section{Results and discussion}

Photoluminescence spectra for all measured samples are presented in Fig. 1. The spectrum for sample A consists of two spectrally separated peaks attributed to InAs band gap (0.41 eV) [14] and defect band $(0.38 \mathrm{eV})$ [11]. On the other hand, the spectrum of sample E consists of solely one wide peak arising from band gap, broadened by Burstein-Moss effect. It is worth noting that we do not observe Mahan exciton [9], since we do not observe any peak higher than band gap energy, so it cannot interfere with Burstein-Moss broadening.
Although the concentration for all samples is much higher than $1 \times 10^{14} \mathrm{~cm}^{-3}$ (Mott transition in InAs), we observe Burstein-Moss broadening only for the two most heavily doped samples: $\mathrm{D}\left(2 \times 10^{17} \mathrm{~cm}^{-3}\right)$ and $\mathrm{E}\left(3 \times 10^{18} \mathrm{~cm}^{-3}\right)$. It is in agreement with previous calculations [13].

In the presented spectra we observe no correlation between intensity of defect peak and samples quality obtained from the XRD measurements. For sample C, Full Width at Half Maximum (FWHM) of the XRD peak is higher than for sample B (Table 1), while the intensity of photoluminescence defect peak is much lower. Moreover, for sample $D$, in spite of the highest FWHM value of the XRD peak (Table 1), there is no defect peak observed. We suspect the intensity of defect peak decreases rapidly with increasing concentration (above $1 \times 10^{16} \mathrm{~cm}^{-3}$ ). Similar effect was observed for the low doped p-type GaSb samples [18]. However, in that case the defect peak was created by acceptor-donor interaction while Fermi level was inside band gap or even below valence band [18]. In our experiments Fermi level is above conduction band and the defect states are $35 \mathrm{meV}$ above the valence band [11]. All those details suggest that the defect peak intensity decrease mechanisms for InAs:Si and GaSb samples are different.

One of the possible explanations may be the appearance of non-zero momentum electrons that take part in creation of band gap peak, whereas the defect peak is formed due to defect states which possess very low momentum. If defect states momentum is close to zero, then for low-doped sample in which almost all electrons have zero momentum there are many electrons that may recombine into defect states. If the concentration is increased, there are much fewer electrons with zero momentum and much more with higher momentum [13]. In this case, electrons have much lower probability to recombine into the defect states.

The disappearance of the defect peak is also observed with increasing temperature (Fig. 2). For samples A and B defect peak intensity is the highest at $20 \mathrm{~K}$ and disappears at $80 \mathrm{~K}$ and $100 \mathrm{~K}$, respectively. In this case, there is no BursteinMoss shift, but instead Fermi-Dirac distribution 


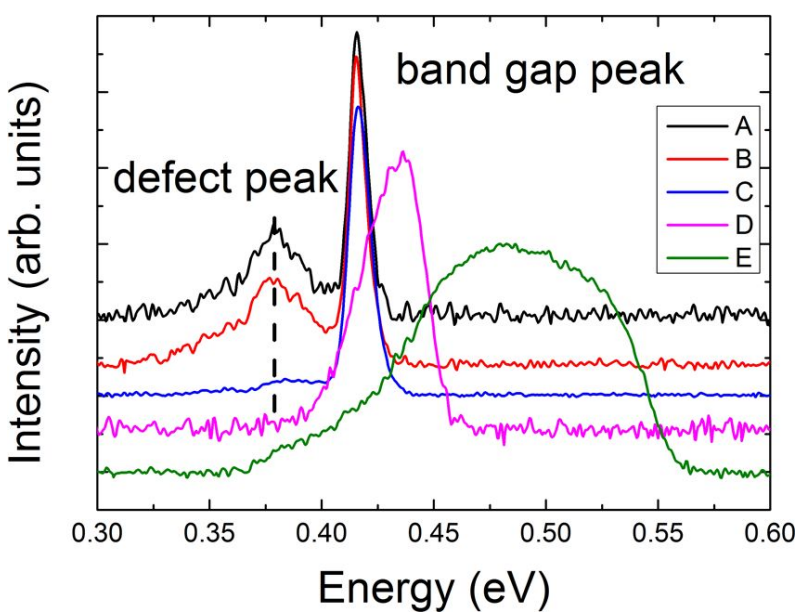

Fig. 1. Photoluminescence spectra for all samples, measured at $20 \mathrm{~K}$.

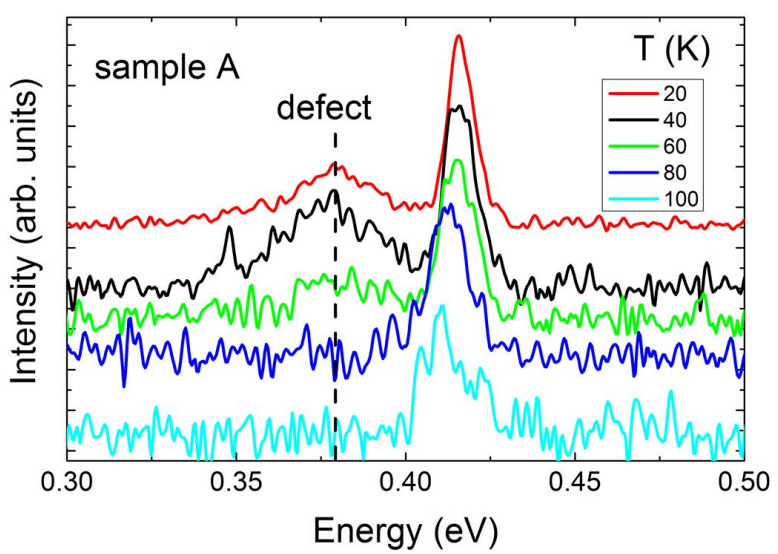

(a)

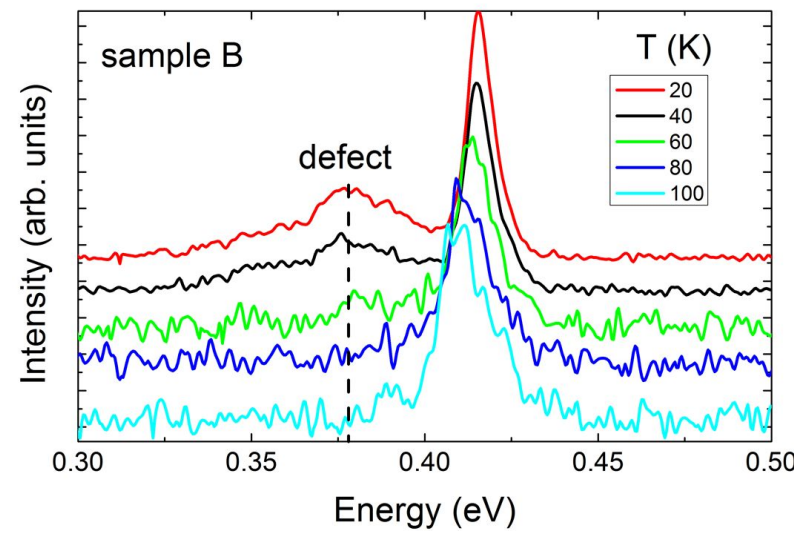

(b)

Fig. 2. Photoluminescence spectra for samples A (a) and $\mathrm{B}$ (b) obtained in the temperature range from $20 \mathrm{~K}$ to $100 \mathrm{~K}$. flattening takes place. It may cause the increase of the amount of electrons with higher energy and momentum. However, there are also other effects that shift the position of band gap peak to lower energies [12]. Thus, it is difficult to conclude whether the momentum of electrons is higher or not. Other possible explanation is that the energy dispersion at high temperature is stronger for defect states than for the bottom of conductive band and the top of valence band.

\section{Conclusions}

Presented results confirm previous calculations which predict the appearance of Burstein-Moss broadening only for InAs samples doped higher than $1 \times 10^{17} \mathrm{~cm}^{-3}$. We have also shown the decrease of the defect peak intensity with both increasing concentration (taking place above Mott transition) and increasing temperature.

\section{Acknowledgements}

We acknowledge support by The National Science Centre: the Grant No. OPUS/UMO-2015/19/B/ST7/02200 and PBS 653.

\section{References}

[1] PARry M.K., Krier A., Electron. Lett., 23 (1994), 1968.

[2] Dobbelaere W., Boeck de J., Hermemans P., Mertens R., Borghs G., Luyten W., LANduYt VAN J., Appl. Phys. Lett., 7 (1992), 868.

[3] Dobbelaere W., Raedt De W., Boeck DE J., Mertens R., Borghs G., Electron. Lett., 4 (1992), 372.

[4] Lin R.M., Tang S.F., Lee S.C., Kuan C.H., Chen G.S., Sun T.P., Wu J.C., IEEE T. Electron. Dev., 9 (1997), 209.

[5] Sun W., Lu Z., Zheng X., Campbell J.C., MadDOX S.J., NAIR H.P., BANK S.R., IEEE J. Quantum Elect., 2 (2013), 154.

[6] Sandalla I.C., Bastiman F., White B., Richards R., MEndes D., David J.P.R., TENLESS C.H., Appl. Phys. Lett., 17 (2014), 171109.

[7] Maddox S.J., Sun W., LU Z., NAIR H.P., CAMPBell J.C., BANK S.R., Appl. Phys. Lett., 10 (2012), 151124.

[8] Ker P.J., David J.P.R., TAN C.H., Opt. Express, 28 (2012), 29568.

[9] Fuchs F., Kheng K., Koidl P., Sschwarz K., Phys. Rev. B, 11 (1993), 7884.

[10] Cardona M., Phys. Rev., 3 (1961), 752. 
[11] LacroiX Y., Tran C.A., Watkins S.P., WaLT M.L.W., J. Appl. Phys., 11 (1996), 6416.

[12] VARshni Y.P., Physica, 1 (1967), 149.

[13] VILKOTSKII V.A., DOMANEVSKII D.S. KaKanakov R.D., KRASOVSKII V.V., TKaCheV V.D., Phys. Status Solidi, 1 (1979), 71.

[14] Gladkov P., Nohavica D., ŠoureK Z. Litvinchuk A.P., Iliev M.N., Semicond. Sci. Tech., 4 (2006), 544.

[15] Fisher M., KRIER A., Infrared Phys. Techn., 7 (1997), 405.
[16] Benyahia D., Kubiszyn Ł., Michalczewski K., Keblowski A., Martyniuk P., Piotrowski J., RogalsKi A., Opt. Quantum Electron., 9 (2016), 428.

[17] Motyka M., Sek G., Misiewicz J., Bauer A., Dallner M., Höfling S., Forchel A., Appl. Phys. Express, 12 (2009), 126505.

[18] Wu M.C., Chen C.C., J. Appl. Phys., 9 (1992), 4275.

Received 2017-04-03 Accepted 2017-08-30 